\title{
Determination of Polymorph Structures in Functional Metal Oxides Using Convergent Beam Electron Diffraction
}

\author{
Zanlin Qiu, Abe Owen, Pelagia-Iren (Perena) Gouma and Joerg Jinschek
}

The Ohio State University, Columbus, Ohio, United States

In binary metal oxides (BMO), polymorphic transitions can result in various crystallographic structures which have been shown to exhibit very different and distinct physical and chemical properties. Thus, exact structural determination is essential as these changes in their crystal structures offer fine control over a wide variety of different properties and, therefore, open up a wide field of applications. However, distinguishing between different BMO polymorphs is not trivial. A combination of high-resolution X-ray diffraction (XRD), Raman and infrared-ray (IR) spectroscopy might be perform to identify phases. However, this is not a universal approach because strategies for phase identification vary with materials system, and, in the case of BMO polymorphs, might not work at all because of limitations of standard Raman/IR spectrum data of inorganics crystal. Conventional electron-microscopy-based characterization techniques, such as selected area electron diffraction (SAED), nanobeam diffraction (NBE), highresolution TEM (HRTEM), high-resolution STEM (HRSTEM), provide information that is not always sufficient for polymorph determination, especially in nanocrystalline BMO. A general and universal approach to overcome this and to confirm a specific crystal structure is performing TEM tilt experiments obtaining data from at least 4-6 zone axis orientation (e.g., using SAED patterns, NBE patterns, HRTEM or HRSTEM images). However, this is a complicate experiment and, in case of beam-sensitive materials, this time-consuming approach might not work.

Recent research toward fast and universal phase identification methods shows little progress, but a universal and easy-to-perform method is needed to proceed in the development of functional BMO. Therefore, we propose using Convergent Beam Electron Diffraction (CBED) experiments in one (or a few zone axis orientations) as a direct, easy and universal characterization technique for structure determination of nanocrystalline polymorphic materials. Deficient High Order Laue Zone (HOLZ) lines pattern of different polymorphs (= different space groups) will show distinguishable features. The origin of deficient HOLZ lines is the dispersion surface construction and these line patterns could be theoretically calculated with the help of dynamical scattering theory, enabling accurate determination of accelerating voltages, atomic position, point/space group as well as lattice parameter, ${ }^{[1]}$ as shown in Figure 1 . In order to achieve phase identification, we have to compare experimental and computational deficient HOLZ lines in CBED/large angle CBED (LACBED) /Hollow cone beam CBED (HCB-CBED) patterns. ${ }^{[2]}$

Here, we present results on hafnia $\left(\mathrm{HfO}_{2}\right)$ and tungsten trioxide $\left(\mathrm{WO}_{3}\right)$ nanoparticles with particle size ranging from $50-500 \mathrm{~nm}$. In case of hafnia, there are four published $\mathrm{HfO}_{2}$ polymorphs that can be stabilized under room temperature (RT) and normal pressure. We attempt to identify and distinguish uniquely between the orthorhombic $\mathrm{HfO}_{2}\left(\mathrm{o}-\mathrm{HfO}_{2}\right)$ with space group $\mathrm{Pca} 21$ and tetragonal $\mathrm{HfO}_{2}\left(\mathrm{t}-\mathrm{HfO}_{2}\right)$ phase with space group $\mathrm{P} 42 / \mathrm{nmc}$. The former one exhibits ferroelectric properties while the latter one is a high-permittivity material. ${ }^{[3,4]}$ As for the tungsten trioxide, we attempt to identify hexagonal $\mathrm{WO}_{3}$ (h$\mathrm{WO}_{3}$ ) with space group $\mathrm{P}_{3} / \mathrm{mcm}$, one monoclinic $\mathrm{WO}_{3}\left(-\mathrm{WO}_{3}\right)$ with space group Pc and the other monoclinic $\mathrm{WO}_{3}\left(-\mathrm{WO}_{3}\right)$ with space group $\mathrm{P} 2{ }_{1} / \mathrm{n}$ out of 7 published polymorphs. Those three different $\mathrm{WO}_{3}$ phases show great potential in gas sensor applications, because each polymorph has a distinct response to specific types of gases. For example, $-\mathrm{WO}_{3}$ is a conventional gas sensor that has been used to 
detect $\mathrm{NO}_{\mathrm{x}}, \mathrm{NH}_{3}, \mathrm{H}_{2} \mathrm{~S}$ and $\mathrm{O}_{3} .{ }^{[5,6]}$ Our recent work suggests that h-WO 3 shows good sensing property to Isoprene concentration and $-\mathrm{WO}_{3}$ has high sensitivity to Acetone concentration. ${ }^{[6]}$ Our CBED pattern simulation results in 001 zone axis orientation (Figure 2) suggest that the deficient HOLZ line patterns are unique for the four $\mathrm{HfO}_{2}$ polymorphs, and therefore enable a unique phase identification of $\mathrm{HfO}_{2}$ polymorphs. The results of $\mathrm{WO}_{3}$ polymorphs also support this conclusion. All many-beam CBED patterns are simulated using MBFIT ${ }^{[7]}$. The CBED experiments are performed using a Tecnai 20 TEM at OSU's Center for Electron Microscopy and Analysis (CEMAS).



CBED

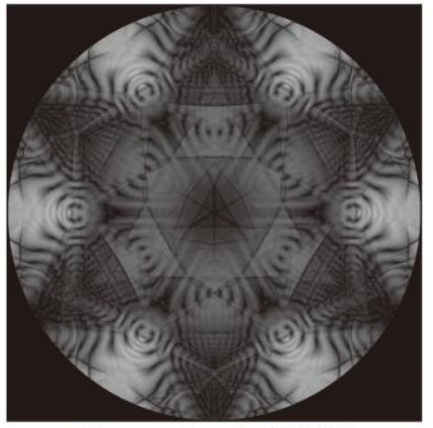

Large Angle CBED

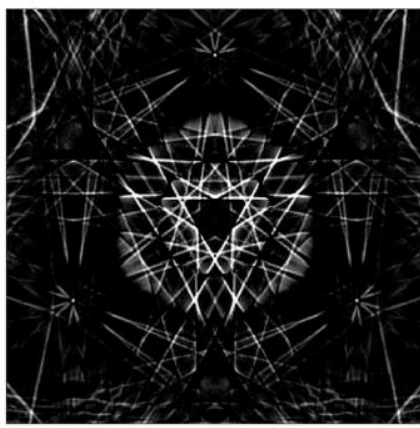

Hollow Cone beam CBED

Analyze High order laue zone (HOLZ) lines pattern with the help of simulation result for precise (with accuracy of $10 \mathrm{pm}$ ) crystal structure determination basing:

Point group, Space group, Lattice parameter, Atomic position



Phases identification of Oxide Polymorphs

Figure 1. Strategy flow chart of phase identification method 

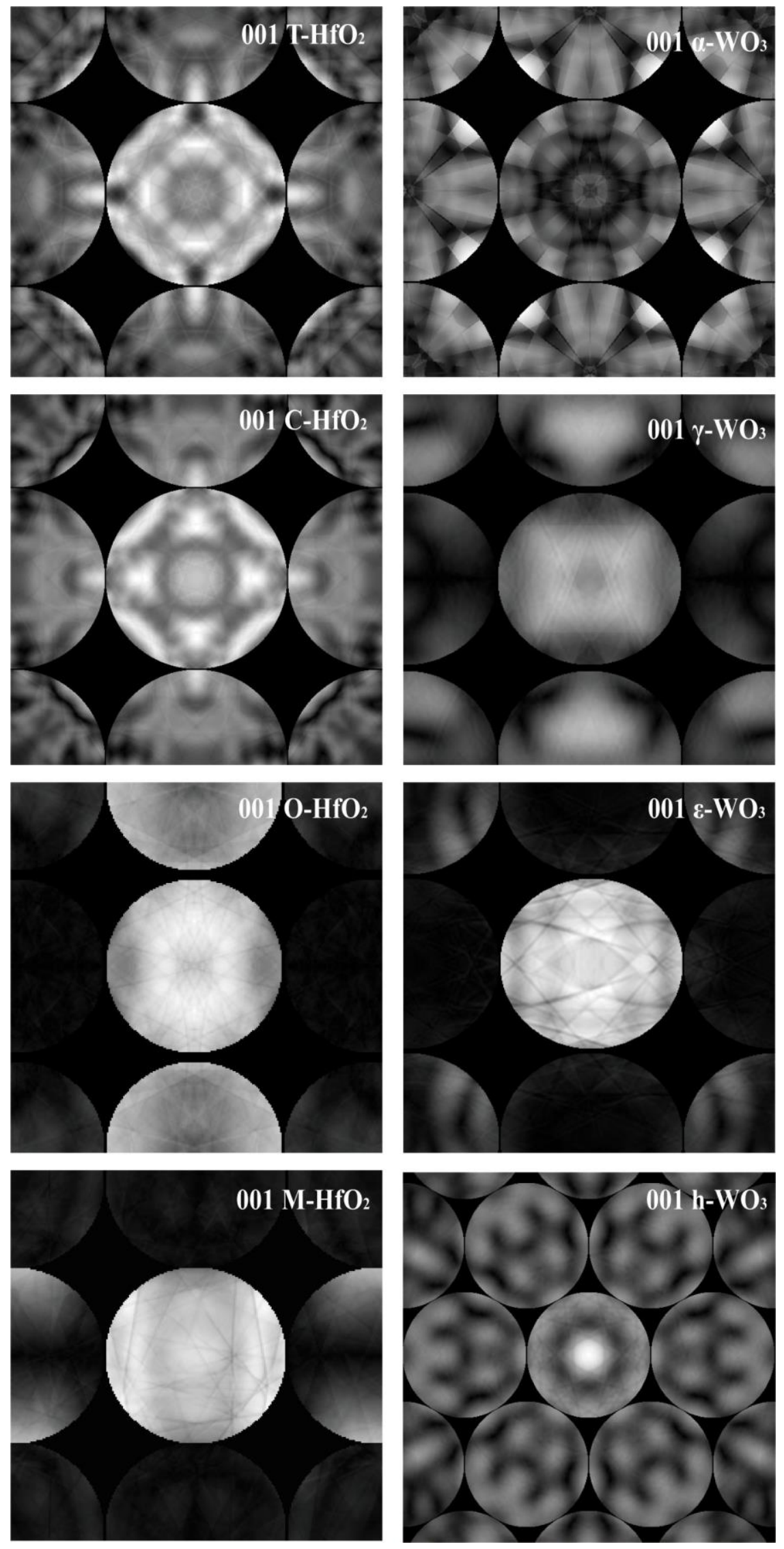
Figure 2. Simulation results of $001 \mathrm{CBED}$ pattern of $\mathrm{HfO} 2$ and $\mathrm{WO} 3$ polymorphs. The $\mathrm{C}, \mathrm{T}, \mathrm{O}$ and $\mathrm{M}-$ HfO2 represents Cubic, Tetragonal, Orthorhombic and Monoclinic Hafnia

\section{References}

[1] Jones PM, Rackham GM, Steeds JW. Higher Order Laue Zone Effects in Electron Diffraction and their Use in Lattice Parameter Determination. Proceedings of the Royal Society of London Series A, Mathematical and Physical Sciences. 1977;354(1677):197-222.

[2] Tanaka M. (1997) Convergent-Beam Electron Diffraction. In: Dorset D.L., Hovmöller S., Zou X. (eds) Electron Crystallography. NATO ASI Series (Series E: Applied Sciences), vol 347. Springer, Dordrecht [3] Böscke TS, et al. Ferroelectricity in hafnium oxide thin films. Applied Physics Letters. 2011;99(10).

[4] Cho D-Y, et al. Stabilization of Tetragonal HfO2 under Low Active Oxygen Source Environment in Atomic Layer Deposition. Chemistry of Materials. 2012;24(18):3534-3543.

[5] Gouma PI. Isoprene sensor/breathalyzer for monitoring sleep disorders. 17th International Meeting on Chemical Sensors - IMCS 20182018. p. 55-56.

[6] Wang L, Teleki A, Pratsinis SE, et al. Ferroelectric WO3 Nanoparticles for Acetone Selective Detection. Chemistry of Materials. 2008 2008/08/01;20(15):4794-4796.

[7] Ogata Y, Tsuda K, Akishige Y, et al. Refinement of the crystal structural parameters of the intermediate phase of h-BaTiO3 using convergent-beam electron diffraction. Acta Crystallographica Section A. 2004;60(6):525-531. 